$\begin{array}{ll}\text { Research Square } & \begin{array}{l}\text { Preprints are preliminary reports that have not undergone peer review. } \\ \text { They should not be considered conclusive, used to inform clinical practice, } \\ \text { or referenced by the media as validated information. }\end{array}\end{array}$

\title{
Altered functional connectivity strength in the cuneus and precuneus in patients with persistent postural-perceptual dizziness
}

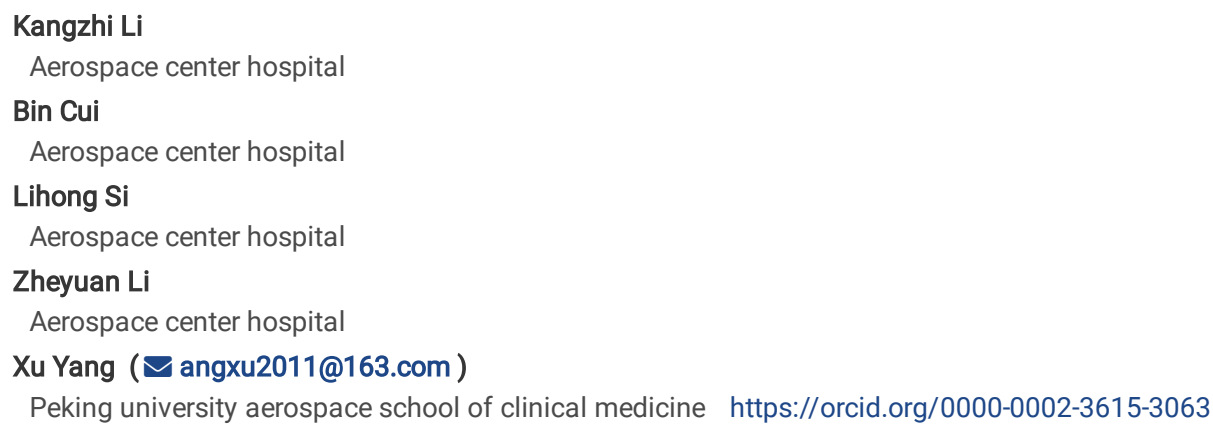

Research

Keywords: persistent-postural perceptual dizziness, resting state functional magnetic resonance imaging, precuneus, cuneus, functional connectivity strength Posted Date: April 14th, 2020

DOI: https://doi.org/10.21203/rs.3.rs-22381/v1

License: (c) (7) This work is licensed under a Creative Commons Attribution 4.0 International License. Read Full License 


\section{Abstract}

Background: Persistent Postural-Perceptual Dizziness (PPPD) is a chronic functional vestibular disorder, but its pathogenesis is still unclear. Results of our previous study revealed altered spontaneous functional activity of cuneus and precuneus in PPPD patients, which may be associated with the development of PPPD. The study aimed to explore the changes in functional integration, especially the changes in functional connectivity strength (FCS) in the cuneus and precuneus in patients with PPPD using FCS and seed-based functional connectivity (FC) analyses.

Methods: Twelve PPPD patients and 12 healthy controls were enrolled. Functional MRI (fMRI) was further performed in all subjects. Global FCS, long-range FCS (IFCS) and short-range FCS (sFCS) were calculated to explore the FCS changes in PPPD patients. Moreover, seed-based FC analysis was performed on regions with altered FCS to further investigate the alterations in FC and the possible causes for FCS changes. Correlation analysis between FCS/FC and clinical indicators in PPPD patients were performed.

Results: Compared with healthy controls, no significant change in global FCS and IFCS was found in PPPD patients. PPPD patients exhibited weaker sFCS in the cuneus and precuneus ( $K=621, \mathrm{P}<0.05$, FWE corrected). Areas showing weaker sFCS included precuneus, cuneus, extending inward along the parietooccipital sulcus, covering parahippocampal gyrus and cortices at the splenium of the corpus callosum. Further seed-based FC analysis showed reduced FC with precuneus, cuneus, indicating that weaker SFCS was mainly caused by reduced FC in the precuneus and cuneus. Changes in sFCS and FC were negatively correlated with DHI and DHI-F scores, and the lower the FCS values, the more severe the subjective symptoms of patients.

Conclusion: PPPD patients exhibited altered FCS in the bilateral precuneus and cuneus, which may be associated with abnormal integration of visual and vestibular information and abnormal integration between different spatial reference frames.

\section{Background}

Persistent Postural-Perceptual Dizziness (PPPD) is a chronic functional vestibular disorder. This disorder can seriously affect the patient's daily activities and quality of life [1], But the exact pathogenesis of PPPD is still unclear.

Exploring the pathophysiological mechanism of PPPD is essential to investigate the nature of this disease, and can provide the basis for exploring new treatments for PPPD. In recent years, the functional magnetic resonance imaging (fMRI) has been the main method for measuring brain function changes and pathophysiological mechanisms of PPPD [2-5]. Previous studies of PPPD mainly focused on two aspects, i.e. brain's response to task stimuli and changes in brain functional activity during resting state, and revealed brain function changes in patients with PPPD, but there was high heterogeneity among studies, which may be related to the difference in study design and subjects included. The previous studies not only included patients with PPPD, but also included patients with visual induced dizziness (VID) and chronic subjective dizziness (CSD) [2-5]. Although the definition of PPPD was derived from the four precursors, including PPV, SMD, VID, and CSD, but there are differences in the diagnostic criteria of the four precursors and PPPD [1]. The results of previous study need to be further validated in patients who met the diagnostic criteria of PPPD. In our previous study, we included PPPD patients without peripheral vestibular lesions, the results showed that the spontaneous functional activity of cuneus and precuneus in PPPD patients were altered, FC between cuneus and precuneus, as well as between the precuneus and precentral gyrus were decreased, which indicated that cuneus and precuneus may be the responsibility lesions of PPPD [6]. Based on the findings of previous study, investigating the changes in functional integration in the brain of patients with PPPD, especially the functional connectivity patterns changes in the cuneus and precuneus will help us further explain the pathophysiological mechanism of PPPD.

It should be noted that in previous FMRI studies of PPPD, FC analysis was performed using a seed-region based approach seeds or automated anatomical labeling (AAL) template based on prior anatomical knowledge or partitions, but these methods neglect the changes in the entire brain and may be affected by subjective factors and template selection, which may also cause high heterogeneity between studies. Functional connectivity strength (FCS) analysis is a voxel-based data-driven method to examine the functional hubs and connectivity patterns in brain networks [7-9]. Unlike the above mentioned of traditional FC analyses, FCS can measure the number of functional connections per voxel, and voxel with higher FCS values indicate a larger number of functional connections between this voxel with other voxels, sugggesting stronger ability of information transfer. Moreover, FCS can be classified into short-range FCS (sFCS) or long-range FCS (IFCS) according to the anatomical distance of a given voxel. Therefore, the FCS can determine the functional nodes in the cortex and subcortical regions without selecting any seed regions, and avoid heterogeneity observed across different anatomical templates. However, based on FCS analysis, we are unable to determine the possible causes for FCS changes. FCS combined with seed-based FC analysis can help us to explore functional connectivity patterns changes in patients with PPPD.

Given the above background, and based on our previous study, we used FCS and seed-based FC analysis in the present study to explore the changes in functional integration, and further elucidate functional abnormalities within brain regions in patients with PPPD.

\section{Subjects And Methods}

All subjects volunteered to participate in this study and signed informed consent. This study was approved by the Ethics Committee of Peking University Aerospace School of Clinical Medicine, China.

\section{Subjects}

A total of 12 patients with PPPD who received treatment in our hospital from January 2018 to December 2018 were included in the study. Detailed medical information of all patents was collected. The symptoms of all patients were evaluated by dizziness handicap inventory score (DHI). The diagnosis of PPPD was based on the diagnostic criteria of Bárány Society (the 2017 edition). 12 age- and gender-matched healthy controls were also included. All healthy controls had no history of headache or dizziness. fMRI was performed in all subjects.

Page 2/9 
All MRI scans were performed on 3.0-Tesla MR scanner (MAGNETOM Skyra syngo MR D13; Siemens, Germany) with a 32-channel head and neck coil. fMRI data were acquired according to the method of our previous study [6]. fMRI data were processed with DPABI software on the MATLAB2013 platform [10].

\section{fMRI data preprocessing}

The acquired images were processed using Statistical Parametric Mapping (SPM12). Data were converted from DICOM format to NIFTI. The first 10 time points of the functional images were deleted due to the possible instability of the initial MRI signal. Then slice timing and realignment were conduct. To ensure the accuracy of the position information, and the subjects who had more than $1.5 \mathrm{~mm}$ head translation in $\mathrm{x}, \mathrm{y}$, or $\mathrm{z}$ and $1.5^{\circ}$ head rotation were removed. Then using DARTEL, fMRI images were registered and spatially normalized to Montreal Neurological Institute space. Furthermore, a linear regression model was used to remove the interference signal in the blood oxygen level dependent (BOLD) signal. At last, data was band-pass filtered in the range $0.01-0.1 \mathrm{~Hz}$ to reduce low-frequency drift and physiological high-frequency noise.

\section{FCS calculation}

For each subject, Pearson's correlation coefficients were calculated between the time series of each voxel and the other voxels within a whole brain gray matter mask, and a whole-brain functional connectivity matrix was constructed for each subject. According to previous study, a threshold of 0.2 was chosen to eliminate voxels with weak correlations due to signal noise. To improve normality, the FC matrix was transformed to a z-score matrix using a Fisher's z transformation [11]. FCS for a given voxel was calculated as the summation of the connectivity strength between this voxel and all the other voxels. In order to further examine the effects of anatomical distance on FCS analyses, FCS was divided into sFCS and IFCS, the sFCS is defined as the sum of the correlations between the given voxel and other voxels with an anatomical distance less than $75 \mathrm{~mm}$, while the IFCS is defined as the sum of the connections with an anatomical distance greater than $75 \mathrm{~mm}$ [12]. The long- and short-range matrix was transformed to a z-score matrix using a Fisher's z transformation. Finally, images were spatially smoothed using Gaussian kernel with full-width at half-maximum of $8 \mathrm{~mm}$. In order to further verify the reproducibility of our result, we also used two additional correlation thresholds $(0.15,0.3)$ to compute the FCS maps.

\section{Seed-based FC analysis}

To further examine the possible causes for FCS changes in PPPD patients, a seed-based FC analysis was conducted. Seed regions was established as region of interest (ROIs) as $6 \mathrm{~mm}$ radius spheres centered on at the peak coordinate of each cluster showing significant changes in global, long- or short-rang FCS. Pearson's correlation coefficients were calculated between the time series of the seed ROI with those of all voxels within the gray matter mask. A Fisher's z transformation was further applied to improve the normality of the correlation coefficient. Finally, images were spatially smoothed using Gaussian kernel with full-width at half-maximum of $8 \mathrm{~mm}$.

\section{Statistical analysis}

The differences in the results of FCS and Seed-based FC analysis between PPPD patients and healthy subjects were tested using a two-sample T test based SPM12 software with age and gender as covariants. $\mathrm{P}<0.05$ was considered to be statistically different. Multiple comparisons corrections were applied using a family-wise-error correction (FWE). MASK was defined as brain region with abnormal FCS, and region of interest (ROIs) was defined as 6 mm radius spheres centered on the peak coordinate of each cluster showing significant FCS/FC changes. Correlation analysis was conducted between FSC/FC and clinical baseline data in patients with PPPD. The P value of less than 0.05 was considered to indicate a statistically significant difference.

\section{Results}

\section{Clinical data}

Twelve PPPD patients, including 8 males and 4 females were included. All participants were right-handed. The detailed clinical baseline data were shown in Table 1

\section{FCD changes in patients with PPPD}

Compared with healthy controls, no significant change in global FCS and IFCS was found in PPPD patients, sFCS in the cuneus and precuneus was reduced in PPPD patients ( $K=621, P<0.001$, FWE corrected). Areas showing reduced sFCS included left precuneus on the left side, the cuneus and precuneus, extending inward along the parieto-occipital sulcus, covering parahippocampal gyrus and cortices at the splenium of the corpus callosum on the right side (Figure 1).

\section{Validation Results}

The reproducibility of our results were further verified with the FCS analysis at the correlation thresholds of 0.15 and 0.3 . FCS analysis with correlation thresholds of 0.15 and 0.3 revealed reduced sFCS in cuneus and precuneus in PPPD patients, which was consistent with the results calculated using a threshold of 0.2 (Figure 2,3).

\section{Results of seed-based FC analysis in patients with PPPD}

Seed-based FC analysis was further performed to investigate the functional alterations in patients with PPPD. Twelve brain regions with reduced sFCS were selected as seeds (Table 2). The results showed weaker FC with right precuneus and cuneus, indicating that reduced sFCS was mainly due to the weaker FC in the right precuneus and cuneus (Table 3 ). 


\section{Correlation analysis}

FCS changes in the precuneus and cuneus, FC changes in ROI2-cluster1, ROI5-cluster1, ROI12-cluster1 were negatively correlated with the DHI score. Changes in FC in ROI4-cluster1, ROI5-cluster3, ROI12-cluster14 were negatively correlated with DHI-F and DHI-E scores in PPPD patients (Table 4).

\section{Discussion}

In our study, FCS analysis revealed that SFCS in the bilateral precuneus and cuneus was decreased in patients with PPPD, and no significant alterations was observed in global FCS and IFCS in PPPD patients, the results indicated that FCS changes was mainly occurred in the cuneus and precuneus. Results of seedbased FC analysis on regions with reduced FCS showed that reduced SFCS was mainly caused by the weakened FC in the right precuneus and cuneus. Moreover, FCS/FC changes were negatively correlated with clinical symptom scores, and the lower the FCS/FC values, the more severe the patients' symptoms.

Our previous study performed local brain functional analysis in PPPD patients, and found that the amplitude of low-frequency fluctuation (ALFF) and regional homogeneity $(\mathrm{ReHo})$ in the right precuneus were significantly decreased, indicating that the spontaneous functional activity in this area was altered [6]. In the current study, results of FCS further confirmed the dysfunction of the precuneus, and the areas with reduced FCS were more extensive than areas with abnormal ALFF and ReHo, indicating that precuneus dysfunction in PPPD patients may be more obvious compared with the results from our previous study. Margulies et al performed FC analysis of the precuneus using resting-state functional MRI, and found three subregions within the precuneus, including anterior sensorimotor, central cognitive, and posterior visual regions [13]. In our study, the altered FCS in the precuneus was mainly located in the posterior visual region, which is mainly involved in the integration of vestibular and visual information. For patients with PPPD, the reduced sFCS in the precuneus may lead to abnormal integration of vestibular and visual information, causing abnormal spatial perception, and resulting in dizziness in PPPD patients. In addition, the higher the FCS values, the greater the connection strength of the node to other nodes. The precuneus is the important network node within the default mode network (DMN) [14]. It is speculated that the dysfunction of the precuneus may lead to abnormal function of DMN, which cannot effectively evaluate the information from internal and external environment, and cannot achieve a clear spatial perception [15]. If abnormality in the DMN persists during rest state, persistent dizziness and unsteadiness may occur in patients with PPPD.

In our study, the reduced sFCS of the right precuneus in PPPD patients was mainly located at the junction of the parietooccipital and calcarine sulci, which mainly include the cortex around the calcarine sulcus, posterior cingulate cortex, and retrosplenial cortex. The cortex around the calcarine sulcus is mainly located in the visual association cortex, which is involved in visual information processing and the integration of vision and other information [16]. Functional abnormalities in this area may lead to abnormalities in visual information processing and integration of visual and vestibular information, resulting in abnormal spatial perception, causing symptoms such as dizziness and unsteadiness in patients with PPPD. Functional abnormalities in this area may be related to the aggravation of dizziness and unsteadiness that triggered by complex visual stimuli. When patients are exposed to visual stimuli or complex visual environments, the complex visual information is conveyed which exacerbates the deficits in visual information processing and integration, and aggravate the symptoms (dizziness and unsteadiness) of PPPD patients.

Studies have shown that the posterior cingulate cortex plays an important role in emotion regulation, spatial attention, memory, and navigation [17-20]. The abnormal function of the posterior cingulate cortex in patients with PPPD may cause abnormalities in the functions of spatial attention and navigation in PPPD patients, leading to abnormal spatial perception when patients expose to complex visual environment, and resulting in the occurrence of dizziness. The posterior cingulate cortex is also an important part of the limbic system and involved in emotion regulation. The abnormal function of this area may also lead to emotion regulation abnormalities in PPPD patients, which may be associated with the different levels of anxiety and depression in PPPD patients.

Studies have shown that the retrosplenial cortex directly participates in the coordination and transformation between the egocentric and allocentric spatial reference frames, that enables us to orient ourselves in the environment [21-26]. Patients with retrosplenial cortex lesions are unable to identify landmarks, but are unable to use this information to derive navigational information orientate themselves $[27,28]$. The abnormal function of the retrosplenial cortex in patients with PPPD may cause deficits in the coordination of egocentric and allocentric spatial reference frames, leading to abnormal spatial perception and spatial disorientation, resulting in dizziness in patients with PPPD.

Seed-based FC analysis was further conducted to explore FC changes in regions with reduced FCS, and decreased FC was found in the same regions showing reduced FCS, the results indicated that decreased FCS was caused by the decreased FC in the precuneus and cuneus, which further confirmed the local functional abnormality of the precuneus and cuneus. It is worth noting that in seed-based FC analysis, although most of the ROIs showed decreased FC with precuneus and cuneus, but some ROIs showed altered FC with other regions. ROI3, ROI7, ROI12 showed weaker FC with the cerebellum, ROI3, ROI12 showed weaker FC with precentral gyrus and postcentral gyrus. The results indicated that functional abnormalities in precuneus and cuneus in patients with PPPD can cause abnormal sensorimotor integration, and weakened ability to control body movement and posture through integrating visual and vestibular information, thereby causing unsteadiness in patients with PPPD.

In addition, Kaoru et al. [29] showed that various sensory information including visual, vestibular or proprioceptive sensations is routed to the central cortex, and integrated by posterior parietal cortex and temporoparietal cortex to create an internal estimate model of body, then sensory information is further transfered to premotor and supplementary motor areas for movement encoding, which can produce voluntary movements and maintain body balance. This transformation of sensory information can also be called dorsal stream. In patients with PPPD, altered functional activity of the precuneus and cuneus cause abnormal information exchange in cuneus-precuneus, precuneus- premotor area/precentral gyrus. We speculate that the dorsal stream, i.e. cuneus-precuneuspremotor area is abnormal, causing symptoms such as dizziness and instability in PPPD patients.

\section{Conclusion}


Altered FCS in the precuneus and cuneus in patients with PPPD may lead to abnormal integration of visual and vestibular information, which may be associated with persistent dizziness, instability and exacerbations of those symptoms with exposure to complex visual stimuli in PPPD patients.

\section{Abbreviations}

FCS, functional connectivity strength

PPPD, persistent-postural perceptual dizziness

fMRI, functional magnetic resonance imaging

IFCS, long-range FCS

sFCS, short-range FCS

ROIs, region of interest

FWE correction, family-wise-error correction

DMN, default mode network

ReHo, regional homogeneity

ALFF, amplitude of low-frequency fluctuation

VID, visual induced dizziness

CSD, chronic subjective dizziness

\section{Declarations}

\section{Authors' Contributions}

$\mathrm{XY}$ contributed to the conception and design of the study; $\mathrm{KL}, \mathrm{BC}, \mathrm{LS}, \mathrm{ZL}$ collected the clinical data and analysed the results; $\mathrm{KL}$ drafted and corrected the manuscript. All authors read and approved the final manuscript.

\section{Acknowledgements}

The study was supported by Aerospace Center Hospital (Grant No. YN201912).

\section{Competing interests}

The authors declare that they have no competing interests.

\section{Availability of data and materials}

Not applicable.

\section{Ethics approval and consent to participate}

This study was approved by the Ethics Committee of Peking University Aerospace School of Clinical Medicine, China. Informed, written consent was obtained from all individual participants included in the study.

\section{References}

1. Staab JP, Eckhardt-Henn A, Horii A, Jacob R, Strupp M, Brandt T, Bronstein A. Diagnostic criteria for persistent postural-perceptual dizziness (PPPD): Consensus document of the committee for the Classification of Vestibular Disorders of the Barany Society. J Vestib Res. 2017;27(4):191-208.

2. Zang YF, He Y, Zhu CZ, Cao QJ, Sui MQ, Liang M, Tian LX, Jiang TZ, Wang YF. Altered baseline brain activity in children with ADHD revealed by restingstate functional MRI. Brain Dev. 2007; 29(2):83-91.

3. Lee JO, Lee ES, Kim JS, Lee YB, Jeong Y, Choi BS, Kim JH, Staab JP. Altered brain function in persistent postural perceptual dizziness: A study on resting state functional connectivity. Hum Brain Mapp. 2018; 39(8):3340-3353.

4. Van Ombergen A, Heine L, Jillings S, Roberts RE, Jeurissen B, Van Rompaey V, Mucci V, Vanhecke S, Sijbers J, Vanhevel F, Sunaert S, Bahri MA, Parizel PM, Van de Heyning PH, Laureys S, Wuyts FL. Altered functional brain connectivity in patients with visually induced dizziness. Neuroimage Clin.2017;14:538545.

5. Riccelli R, Passamonti L, Toschi N, Nigro S, Chiarella G, Petrolo C, Lacquaniti F, Staab JP, Indovina I. Altered Insular and Occipital Responses to Simulated Vertical Self-Motion in Patients with Persistent Postural-Perceptual Dizziness. Front Neurol. 2017;8:529. 
6. Li K, Si L, Cui B, Ling X, Shen B, Yang X. Altered spontaneous functional activity of the right precuneus and cuneus in patients with persistent posturalperceptual dizziness. Brain Imaging Behav. 2019 Jul 16. doi: 10.1007/s11682-019-00168-7. [Epub ahead of print]

7. Buckner RL, Sepulcre J, Talukdar T, Krienen FM, Liu H, Hedden T, Andrews-Hanna JR, Sperling RA, Johnson KA. Cortical hubs revealed by intrinsic functional connectivity: mapping, assessment of stability, and relation to Alzheimer's disease. J Neurosci.2009;29(6):1860-1873.

8. Liang X, Zou Q, He Y, Yang Y. Coupling of functional connectivity and regional cerebral blood flow reveals a physiological basis for network hubs of the human brain. Proc Natl Acad Sci U S A. 2013;110(5):1929-1934.

9. Dai Z, Yan C, Li K, Wang Z, Wang J, Cao M, Lin Q, Shu N, Xia M, Bi Y, He Y. Identifying and Mapping Connectivity Patterns of Brain Network Hubs in Alzheimer's Disease. Cereb Cortex. 2015;25(10):3723-3742.

10. Yan CG, Wang XD, Zuo XN, Zang YF. DPABI: Data Processing \& Analysis for (Resting-State) Brain Imaging. Neuroinformatics. 2016;14(3):339-51.

11. Liu F, Wang Y, Li M, Wang W, Li R, Zhang Z, Lu G, Chen H. Dynamic functional network connectivity in idiopathic generalized epilepsy with generalized tonic-clonic seizure. Hum Brain Mapp. 2017;38(2):957-973.

12. Liu F, Zhu C, Wang Y, Guo W, Li M, Wang W, Long Z, Meng Y, Cui Q, Zeng L, Gong Q, Zhang W, Chen H. Disrupted cortical hubs in functional brain networks in social anxiety disorder. Clin Neurophysiol. 2015;126(9):1711-1716.

13. Margulies DS, Vincent JL, Kelly C, Lohmann G, Uddin LQ, Biswal BB, Villringer A, Castellanos FX, Milham MP, Petrides M. Precuneus shares intrinsic functional architecture in humans and monkeys. Proc Natl Acad Sci U S A. 2009;106(47):20069-20074.

14. Utevsky A V, Smith D V, Huettel S A. Precuneus is a functional core of the default-mode network. J Neurosci. 2014; 34(3):932-940.

15. Raichle M E. The brain's default mode network. Annu Rev Neurosci. 2015;38:433-447.

16. Waberski TD, Gobbelé R, Lamberty K, Buchner H, Marshall JC, Fink GR. Timing of visuo-spatial information processing: electrical source imaging related to line bisection judgements. Neuropsychologia. 2008; 46(5):1201-1210.

17. Boccia M, Sulpizio V, Nemmi F, Guariglia C, Galati G. Direct and indirect parieto-medial temporal pathways for spatial navigation in humans: evidence from resting-state functional connectivity. Brain Struct Funct. 2017;222(4):1945-1957.

18. Hopfinger J B, Buonocore M H, Mangun G R. The neural mechanisms of top-down attentional control. Nat Neurosci. 2000;3(3):284-291.

19. Mesulam MM, Nobre AC, Kim YH, Parrish TB, Gitelman DR. Heterogeneity of cingulate contributions to spatial attention. Neuroimage. 2001;13(6 Pt 1):1065-1072.

20. Bledowski C, Rahm B, Rowe JB. What "works" in working memory? Separate systems for selection and updating of critical information. J Neurosci.2009;29(43):13735-13741.

21. Epstein RA. Parahippocampal and retrosplenial contributions to human spatial navigation. Trends Cogn Sci. 2008;12(10):388-396.

22. Iaria G, Chen JK, Guariglia C, Ptito A, Petrides M. Retrosplenial and hippocampal brain regions in human navigation: complementary functional contributions to the formation and use of cognitive maps. Eur J Neurosci. 2007;25(3):890-899.

23. Mitchell AS, Czajkowski R, Zhang N, Jeffery K, Nelson AJD. Retrosplenial cortex and its role in spatial cognition. Brain Neurosci Adv. $2018 ; 2: 1864918506$.

24. Sherrill KR, Erdem UM, Ross RS, Brown TI, Hasselmo ME, Stern CE. Hippocampus and retrosplenial cortex combine path integration signals for successful navigation. J Neurosci. 2013;33(49):19304-19313.

25. Wilber AA, Clark BJ, Forster TC, Tatsuno M, McNaughton BL. Interaction of egocentric and world-centered reference frames in the rat posterior parietal cortex. J Neurosci. 2014;34(16):5431-5446.

26. Galati G, Pelle G, Berthoz A, Committeri G. Multiple reference frames used by the human brain for spatial perception and memory. Exp Brain Res. 2010;206(2):109-120.

27. Takahashi N, Kawamura M, Shiota J, Kasahata N, Hirayama K. Pure topographic disorientation due to right retrosplenial lesion. Neurology. 1997;49(2):464-469.

28. laria G, Bogod N, Fox CJ, Barton JJ. Developmental topographical disorientation: case one. Neuropsychologia. 2009;47(1):30-40.

29. Takakusaki K. Functional Neuroanatomy for Posture and Gait Control. J Mov Disord. 2017;10(1):1-17.

\section{Tables}

Table 1 Clinical characteristics of PPPD patients 


\begin{tabular}{|c|c|c|c|c|c|c|c|c|c|c|c|c|c|c|c|c|}
\hline \multirow[t]{2}{*}{ Number } & \multirow{2}{*}{$\begin{array}{l}\text { Age } \\
\text { (year) }\end{array}$} & \multirow{2}{*}{$\begin{array}{l}\text { Duration } \\
\text { (month) }\end{array}$} & \multicolumn{3}{|c|}{ Core vestibular symptoms } & \multirow{2}{*}{$\begin{array}{l}\text { Increased } \\
\text { as the day } \\
\text { progressed }\end{array}$} & \multicolumn{3}{|c|}{ Exacerbating factors } & \multirow{2}{*}{$\begin{array}{l}\text { Motion } \\
\text { sickness }\end{array}$} & \multirow{2}{*}{$\begin{array}{l}\text { Fear of } \\
\text { heights }\end{array}$} & \multirow[t]{2}{*}{ DHI } & \multirow{2}{*}{$\begin{array}{l}\text { DHI- } \\
\text { P }\end{array}$} & \multirow{2}{*}{$\begin{array}{l}\text { DHI- } \\
\text { E }\end{array}$} & \multirow{2}{*}{$\begin{array}{l}\text { DHI- } \\
\text { F }\end{array}$} & \multirow[t]{2}{*}{ GAD7 } \\
\hline & & & Dizziness & Unsteadiness & $\begin{array}{l}\text { Non- } \\
\text { spinning } \\
\text { vertigo }\end{array}$ & & $\begin{array}{l}\text { Upright } \\
\text { posture }\end{array}$ & $\begin{array}{l}\text { Active } \\
\text { or } \\
\text { passive } \\
\text { motion }\end{array}$ & $\begin{array}{l}\text { Complex } \\
\text { visual } \\
\text { environment }\end{array}$ & & & & & & & \\
\hline P01 & 35 & 6 & $\sqrt{ }$ & $\sqrt{ }$ & & $\sqrt{ }$ & $\sqrt{ }$ & $\sqrt{ }$ & $\sqrt{ }$ & $\sqrt{ }$ & $\sqrt{ }$ & 40 & 12 & 10 & 18 & 8 \\
\hline P02 & 34 & 3 & $\sqrt{ }$ & $\sqrt{ }$ & $\sqrt{ }$ & $\sqrt{ }$ & $\sqrt{ }$ & $\sqrt{ }$ & $\sqrt{ }$ & $\sqrt{ }$ & & 58 & 18 & 14 & 26 & 5 \\
\hline P03 & 57 & 8 & $\sqrt{ }$ & $\sqrt{ }$ & & & $\sqrt{ }$ & $\sqrt{ }$ & $\sqrt{ }$ & & $\sqrt{ }$ & 62 & 14 & 26 & 24 & 7 \\
\hline P04 & 62 & 120 & $\sqrt{ }$ & $\sqrt{ }$ & & $\sqrt{ }$ & $\sqrt{ }$ & $\sqrt{ }$ & $\sqrt{ }$ & & $\sqrt{ }$ & 65 & 18 & 20 & 28 & 8 \\
\hline P05 & 46 & 156 & $\sqrt{ }$ & $\sqrt{ }$ & & $\sqrt{ }$ & $\sqrt{ }$ & $\sqrt{ }$ & $\sqrt{ }$ & & $\sqrt{ }$ & 52 & 20 & 12 & 20 & 9 \\
\hline P06 & 55 & 36 & $\sqrt{ }$ & $\sqrt{ }$ & & $\sqrt{ }$ & $\sqrt{ }$ & $\sqrt{ }$ & $\sqrt{ }$ & & $\sqrt{ }$ & 56 & 12 & 22 & 22 & 7 \\
\hline P07 & 32 & 12 & $\sqrt{ }$ & $\sqrt{ }$ & & & $\sqrt{ }$ & $\sqrt{ }$ & $\sqrt{ }$ & $\sqrt{ }$ & $\sqrt{ }$ & 50 & 18 & 10 & 22 & 10 \\
\hline P08 & 40 & 6 & $\sqrt{ }$ & $\sqrt{ }$ & & $\sqrt{ }$ & $\sqrt{ }$ & $\sqrt{ }$ & $\sqrt{ }$ & & $\sqrt{ }$ & 52 & 10 & 20 & 22 & 13 \\
\hline P09 & 49 & 7 & $\sqrt{ }$ & $\sqrt{ }$ & & $\sqrt{ }$ & $\sqrt{ }$ & $\sqrt{ }$ & $\sqrt{ }$ & $\sqrt{ }$ & $\sqrt{ }$ & 64 & 18 & 20 & 26 & 9 \\
\hline P10 & 33 & 3 & $\sqrt{ }$ & $\sqrt{ }$ & & $\sqrt{ }$ & $\sqrt{ }$ & $\sqrt{ }$ & $\sqrt{ }$ & & $\sqrt{ }$ & 60 & 12 & 25 & 23 & 7 \\
\hline P11 & 53 & 12 & $\sqrt{ }$ & $\sqrt{ }$ & & $\sqrt{ }$ & $\sqrt{ }$ & $\sqrt{ }$ & $\sqrt{ }$ & $\sqrt{ }$ & & 60 & 13 & 20 & 24 & 7 \\
\hline P12 & 35 & 6 & $\sqrt{ }$ & $\sqrt{ }$ & & $\sqrt{ }$ & $\sqrt{ }$ & $\sqrt{ }$ & $\sqrt{ }$ & & $\sqrt{ }$ & 40 & 12 & 10 & 18 & 9 \\
\hline
\end{tabular}

Table 2 Twelve Regions of interest (ROIs) used as seeds in seed-based functional connectivity analysis

\begin{tabular}{cccccc}
\hline ROIs & Location & X & Y & Z & Radius $\llbracket m m \square$ \\
\hline ROI1 & Cuneus & 18 & -57 & 0 & 6 \\
ROI2 & Cuneus & 12 & -69 & 6 & 6 \\
\hline ROI3 & Cuneus & 12 & -84 & 21 & 6 \\
\hline ROI4 & Cuneus & 12 & -84 & 36 & 6 \\
\hline ROI5 & Precuneus & 18 & -84 & 45 & 6 \\
\hline ROI6 & Precuneus & 6 & -75 & 51 & 6 \\
\hline ROI7 & Precuneus & 0 & -75 & 36 & 6 \\
\hline ROI8 & Precuneus & 0 & -75 & 45 & 6 \\
\hline ROI9 & Precuneus & -3 & -72 & 54 & 6 \\
\hline ROI10 & Precuneus & -15 & -75 & 30 & 6 \\
\hline ROI11 & Precuneus & -3 & -72 & 45 & 6 \\
\hline ROI12 & Cuneus & -3 & -84 & 27 & 6 \\
\hline
\end{tabular}

Table 3 Changes in functional connectivity in the 12 seed ROIs

\begin{tabular}{|c|c|c|c|c|c|c|c|}
\hline \multirow[t]{2}{*}{ ROIs } & \multirow{2}{*}{$\begin{array}{l}\text { Number of } \\
\text { clusters }\end{array}$} & \multirow{2}{*}{$\begin{array}{l}\text { Numbering of } \\
\text { clusters }\end{array}$} & \multicolumn{3}{|c|}{ Positioning } & \multirow{2}{*}{$\begin{array}{l}\text { Number of } \\
\text { voxels }\end{array}$} & \multirow[t]{2}{*}{ Regions } \\
\hline & & & $\mathrm{X}$ & $\mathrm{Y}$ & Z & & \\
\hline \multirow[t]{2}{*}{ ROI1 } & 2 & 1 & 18 & -78 & 42 & 161 & Right precuneus \\
\hline & & 2 & 15 & 9 & -75 & 356 & Left precuneus, cuneus, BA1 \\
\hline \multirow{4}{*}{ ROI2 } & & & & & & & Right precuneus, cortex around the calcarine sulcus \\
\hline & 3 & 1 & 12 & -75 & 12 & 1265 & Bilateral precuneus, cuneus cortex around the calcarine sulcus \\
\hline & & 2 & -24 & 27 & -21 & 108 & Left inferior frontal gyrus \\
\hline & & 3 & -3 & -39 & 51 & 203 & Bilateral paracentral lobule, left posterior insula \\
\hline \multirow{4}{*}{ ROI3 } & 6 & 1 & -24 & 51 & -18 & 139 & Left inferior frontal gyrus, BA11 \\
\hline & & 2 & -21 & -54 & -51 & 271 & Left cerebellum \\
\hline & & 3 & 15 & -57 & -51 & 268 & Right cerebellum \\
\hline & & 4 & -6 & -39 & 54 & 477 & $\begin{array}{c}\text { Bilateral cingulate gyrus, cuneus, paracentral lobule, postcentral } \\
\text { gyrus }\end{array}$ \\
\hline \multirow[t]{2}{*}{ ROI3 } & 6 & 5 & 15 & -75 & 12 & 735 & $\begin{array}{c}\text { Right precuneus, cuneus cortex around the calcarine sulcus, } \\
\text { posterior cingulate gyrus, left cuneus }\end{array}$ \\
\hline & & 6 & 24 & 24 & -27 & 142 & Right inferior frontal gyrus , BA1 \\
\hline ROI4 & 1 & 1 & 21 & -72 & 39 & 1039 & Precuneus, cuneus cortex around the calcarine sulcus \\
\hline \multirow[t]{3}{*}{ ROI5 } & 3 & 1 & 12 & -69 & 6 & 180 & Right precuneus \\
\hline & & 2 & -12 & -87 & 24 & 319 & Left precuneus, cuneus \\
\hline & & 3 & 24 & -84 & 45 & 493 & Right precuneus, cuneus \\
\hline ROI6 & 0 & & & & & & \\
\hline \multirow[t]{2}{*}{ ROI7 } & 2 & 1 & 18 & -66 & 3 & 934 & $\begin{array}{c}\text { Left precuneus, cuneus, right cuneus, cortex around the calcarine } \\
\text { sulcus }\end{array}$ \\
\hline & & 2 & -9 & -60 & -24 & 287 & Left cerebellum, lingual gyrus \\
\hline ROI8 & 0 & & & & & & \\
\hline ROI9 & 1 & 1 & 21 & -48 & 6 & 164 & Cortex around the right calcarine sulcus \\
\hline ROI11 & 1 & 1 & 24 & 6 & 51 & 214 & Bilateral middle frontal gyrus \\
\hline \multirow[t]{4}{*}{ ROI12 } & 4 & 1 & -24 & -57 & 3 & 155 & Cortex around the left calcarine sulcus, lingual gyrus \\
\hline & & 2 & 15 & -60 & -51 & 122 & Right cerebellum \\
\hline & & 3 & -21 & -33 & 54 & 254 & $\begin{array}{c}\text { Left paracentral lobule, precentral gyrus, postcentral gyrus, right } \\
\text { paracentral lobule }\end{array}$ \\
\hline & & 4 & 18 & -75 & 39 & 749 & $\begin{array}{c}\text { Right precuneus, cuneus, cortex around the calcarine sulcus, left } \\
\text { cuneus }\end{array}$ \\
\hline
\end{tabular}


Table 4 Correlation analysis between changes in FCS/FC and dizziness handicap inventory (DHI) score

\begin{tabular}{ccc}
\hline Functional connectivity & DHI & DHI-F \\
\hline sFCS & $\mathrm{r}=-0.605 ; \mathrm{P}=0.037$ & \\
ROI2-cluster1 & $\mathrm{r}=-0.625 ; \mathrm{P}=0.030$ & \\
ROI3-cluster5 & $\mathrm{r}=-0.601 ; \mathrm{P}=0.039$ & $\mathrm{r}=-0.612 ; \mathrm{P}=0.035$ \\
ROI4-cluster1 & $\mathrm{r}=-0.645 ; \mathrm{P}=0.023$ & $\mathrm{r}=-0.682 ; \mathrm{P}=0.015$ \\
\hline ROI5-cluster1 & $\mathrm{r}=-0.640 ; \mathrm{P}=0.025$ & \\
ROI5-cluster3 & $\mathrm{r}=-0.583 ; \mathrm{P}=0.046$ & $\mathrm{r}=-0.604 ; \mathrm{P}=0.038$ \\
ROI12-cluster1 & $\mathrm{r}=-0.650 ; \mathrm{P}=0.022$ & \\
\hline ROI12-cluster4 & $\mathrm{r}=-0.629 ; \mathrm{P}=0.028$ & $\mathrm{r}=-0.618 ; \mathrm{P}=0.032$ \\
\hline
\end{tabular}

\section{Figures}
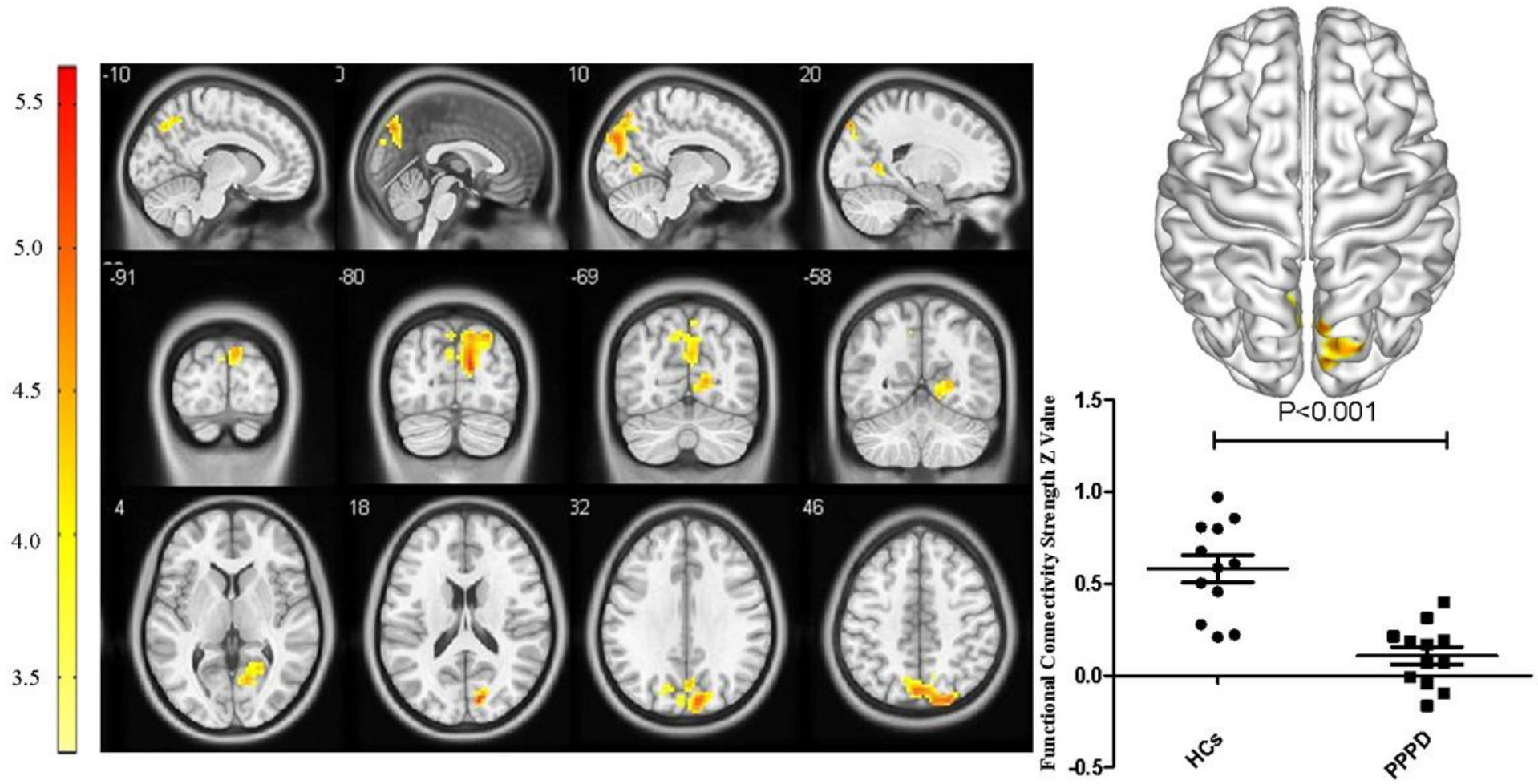

Figure 1

Weaker short-range functional connectivity strength (FCS) in the cuneus and precuneus in patients with PPPD 


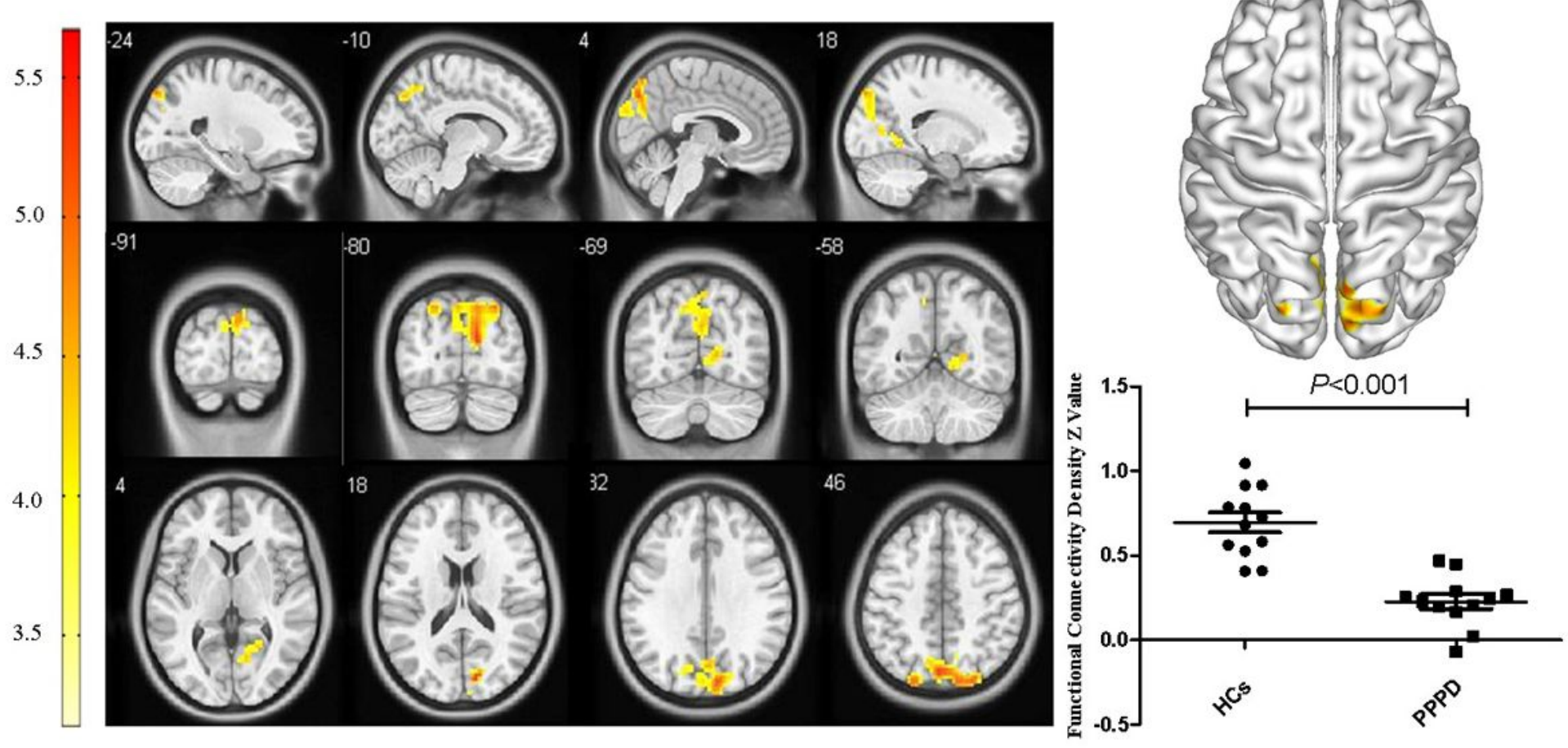

Figure 2

Weaker short-range FCS in the cuneus and precuneus in PPPD patients at a correlation threshold of 0.15

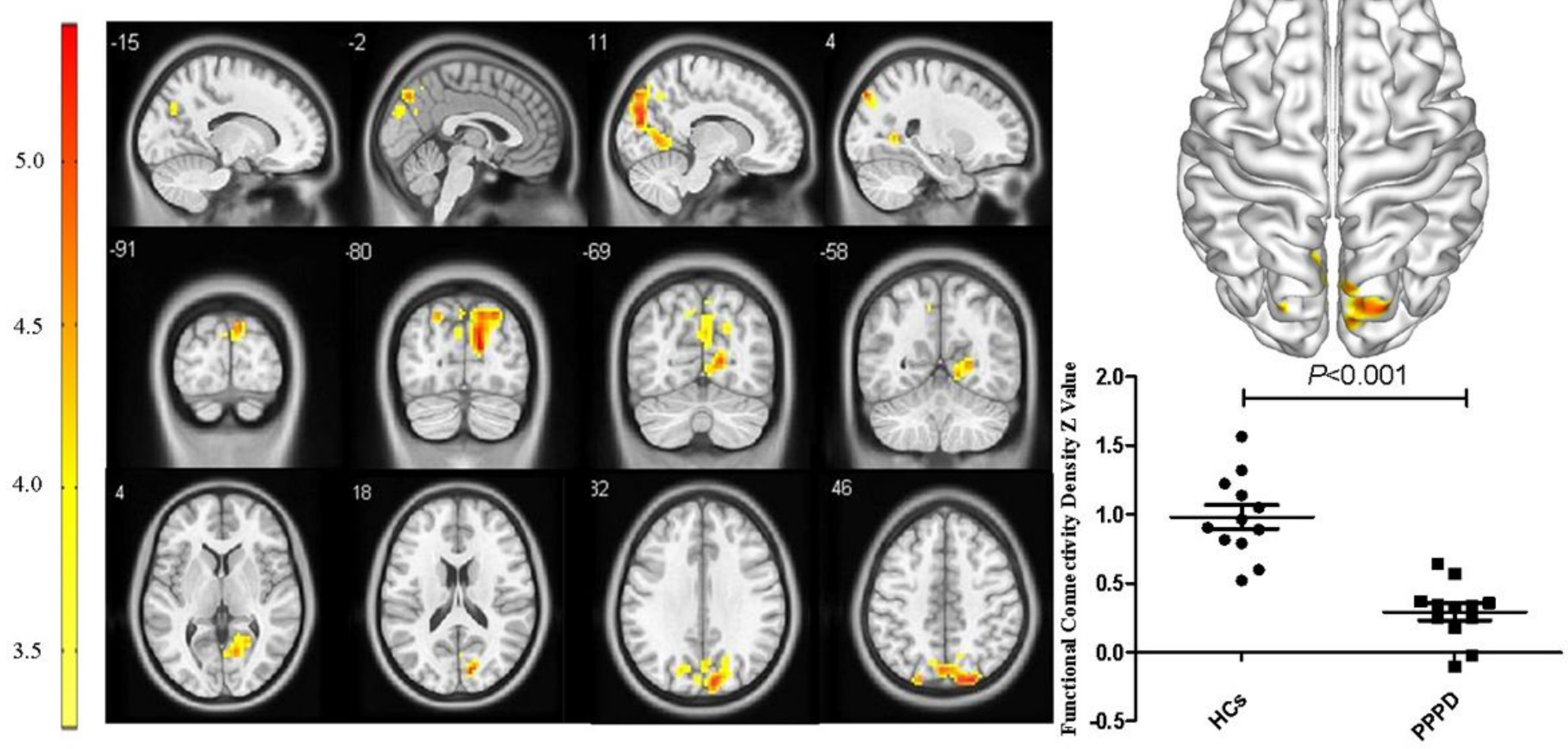

Figure 3

Weaker short-range FCS in the cuneus and precuneus in PPPD patients at a correlation threshold of 0.3 